\title{
Nature's guiding light
}

\author{
The Nobel Prize in Chemistry has been awarded for research inspired by jellyfish. This is a \\ reminder that the natural world continues to hint at solutions to modern technological dilemmas, \\ and that when it comes to simple and effective solutions, nature is usually well ahead of man.
}

The natural world has long inspired poets and artists. But it also serves scientists as a constant signpost towards phenomena yet unknown. If you need testimony to this effect, you need look no further than the 2008 Nobel Prize in Chemistry, awarded to Osamu Shimomura (Marine Biological Laboratory, Massachusetts), Martin Chalfie (Columbia University) and Roger Tsien (University of California, San Diego) for their investigation into a fluorescent protein found in a jellyfish known as a crystal jelly (Aequorea victoria). A denizen of the Pacific Ocean, the crystal jelly is bioluminescent, able to emit green light from an organ around the edge of its umbrella. The protein that enables this green fluorescence, descriptively termed the green fluorescent protein (GFP), was isolated for the first time by Shimomura and his colleagues at the University of Princeton $^{1}$ in 1962 . In the same paper, they also demonstrated that GFP will fluoresce when excited by UV light. This on its own was a worthy advance from a biochemistry point of view. But the real importance of this discovery was brought to the fore when it found an application that changed the way biologists look at cells. Chalfie and his co-workers were able to use GFP to tag other proteins, using its fluorescence properties to track the movement and interaction of otherwise invisible proteins. In 1994, they put this concept into practice, imaging a roundworm (Caenorhabditis elegans), in which touch receptor neurons were tagged and glowed bright green under UV light ${ }^{2}$. Since then, GFP has been used in transgenic experiments, creating fluorescent mice, rabbits and pigs. Shimomura and Chalfie were each awarded a third of the 2008 prize, the final third

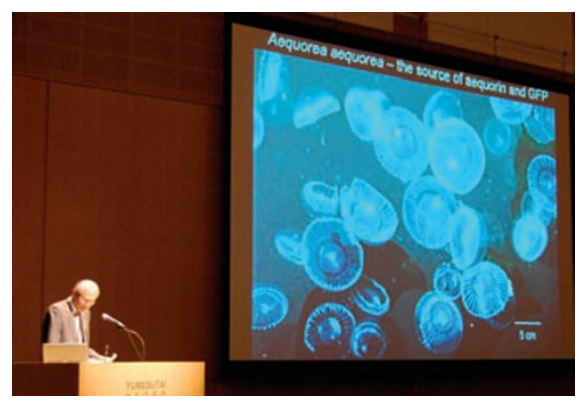

going to Tsien for his development of an entire palette of such fluorescent proteins. Because of the pioneering work of these three men and their colleagues, the use of GFP-like proteins for luminescent marking has now become a standard biological tool.

There are plenty of other examples in photonics of nature taking the lead or putting a physical phenomenon to practical use way before man has cottoned on. Photonic crystals are an often cited example. The iridescent colour of tropical Morpho butterflies is due to a partial photonic bandgap produced because of the pattern of the scales on their wings. Such patterns can now be artificially reproduced in the lab and have potential applications in gas sensing ${ }^{3}$.

Biological structures may also lend a hand in solving a long-standing problem in the application of surfaceenhanced Raman spectroscopy (SERS). Microstructured metal surfaces are known to enhance the Raman scattering from single molecules. Substrates coated in metal nanoparticles have proved to be excellent SERS substrates, but they can't be fabricated reproducibly, hindering their wide-spread adoption in the lab. On the other hand, substrates patterned using the latest microfabrication techniques, such as electron-beam lithography, are expensive. The structures of cicada ${ }^{4}$ and butterfly ${ }^{5}$ wings have been shown to be applicable to SERS when coated with a metal layer. Such substrates are reproducible and can be created quickly and cheaply.

The evolution of the eye, most notably in insects, has also been the source of new technology. The compound lens structure of flies' eyes has been artificially reproduced for use in cameras and medical scopes. Moths' eyes are even more interesting. The surface of their cornea is covered in subwavelength-sized needles. The gradual increase in refractive index deeper beneath the surface dramatically reduces reflection of light incident from all directions ${ }^{6}$, increasing the amount of light that makes it to the optical nerve. Good for the moth, but also useful for producing antireflection coatings. The human eye has also been a good model: its geometry recently inspired the creation of an electronic eye camera ${ }^{7}$. For an overview on nature-inspired photonics, see the excellent review by Andrew Parker and Helen Townley in our sister journal Nature Nanotechnology $y^{8}$.

Nature, it seems, will continue to be a muse for all those who dream, be it in art or in science.

\section{References}

1. Shimomura, O., Johnson, F. H. \& Saiga, Y. J. Cell. Comp. Physiol. 59, 223-239 (1962).

2. Chalfie, M., Tu, Y., Euskirchen, G., Ward, W. W. \& Prasher, D. C. Science 263, 802-805 (1994).

3. Potyrailo, R. A. et al. Nature Photon. 1, 123-128 (2007).

4. Stoddart, P. R., Cadusch, P. J., Boyce, T. M., Erasmus, R. M. \& Comins, J. D. Nanotechnology 17, 680-686 (2006).

5. Moger, J., Cornes, N. L. \& Vukusic, P. Proc. SPIE 6633, 663300 (2007).

6. Clapham, P. B. \& Hutley, M. C. Nature 244, 281-282 (1973). 7. Ko, H. C. et al. Nature 454, 748-753 (2008).

8. Parker, A. R. \& Townley, H. E. Nature Nanotech. 2, 347-353 (2007) 